\title{
Projective Embeddings of Complex Supermanifolds
}

\author{
Claude LeBrun ${ }^{1 \star}$, Yat-Sun Poon ${ }^{2 \star \star}$ and R. O. Wells, Jr. ${ }^{2 \star \star \star}$ \\ ${ }^{1}$ Department of Mathematics, SUNY at Stony Brook, Stony Brook, NY 11794, USA \\ ${ }^{2}$ Department of Mathematics, Rice University, Houston, TX 77001, USA
}

\begin{abstract}
We generalize the Kodaira Embedding Theorem and Chow's Theorem to the context of families of complex supermanifolds. In particular, we show that every family of super Riemann surfaces is a family of projective superalgebraic varieties.
\end{abstract}

\section{Introduction}

In the past few years there has been a great deal of mathematical activity concerning supermanifolds, both real and complex. While much of this work may seem unrelated to the physical motivations of the field, it must be remembered that the subject came into being when physicists realized that it is perfectly consistent to introduce spaces with anti-commuting coordinates [2]; their original train of thought was inextricably linked with the Fermi statistics of quantum field theory, and soon resulted in the formulation of supersymmetric field theories (e.g. $[16,20,26])$. It is therefore perhaps not surprising that the deformation theory of complex supermanifolds has now, with the growing prominence of superstring theory $[9,12]$, proved to be germane to current physics.

The calculation of amplitudes in superstring theory [9] is supposed to involve integration over the moduli space $[6,15]$ of super Riemann surfaces. Unfortunately, this is a rather unruly object, and, in particular, its non-compactness tends to make such integrals ill-defined [23]. On the other hand Mumford [18] has given us a beautiful compactification of the moduli space of Riemann surfaces by treating them as algebraic curves, and Deligne [4] has announced that the same can be done for super Riemann surfaces. In order to carry out such a program, it is first necessary to show that families of complex supermanifolds of dimension $1 / 1$ can always be thought of as families of projective superalgebraic varieties. In this paper,

\footnotetext{
* Research supported in part by NSF grant DMS-8704401

$\star \star$ Research supported in part by NSF grant DMS-4253943

$\star \star \star$ Research also supported in part by NSF grant DMS-4253943
} 
we will explain why this is so, while at the same time deriving results of much greater generality.

A famous result of Whitney [25] asserts that any smooth compact manifold $M^{n}$ can be realized as a submanifold of Euclidean space of $\mathbb{R}^{2 n}$; indeed, there is a canonical embedding of $M$ into the infinite dimensional vector space $\left(C^{\infty}(M)\right)^{*}$ of distributions on $M$, given by

$$
x \mapsto \delta_{x},
$$

where $\delta_{x}$ is the Dirac delta measure centered at $x$, and this gives rise to a finite-dimensional embedding $M \hookrightarrow \mathbf{V}^{*}$ by restricting to almost any subspace $\mathbf{V} \subset C^{\infty}(M)$ of dimension $>2 n$, or by restricting to a somewhat more carefully chosen subspace $\mathbf{V}$ of dimension $2 n$. There is even an easy extension of this to the setting of supermanifolds: a smooth supermanifold $M^{n \mid m}$ can be always be smoothly embedded in $\mathbb{R}^{2 n \mid m+n}$. But for complex manifolds, life is not so simple, because a holomorphic function on a compact complex manifold is necessarily constant (by the maximum principle). A way around this is to look for projective embeddings, for which purpose sections of a line bundle over our complex manifold may be used in place of holomorphic functions. In 1953, using this idea and a powerful cohomological vanishing theorem he had recently proved by Hodge theory and the Bochner method, Kodaira [13] gave an intrinsic characterization of those complex manifolds which can be holomorphically embedded in complex projective space. But several years earlier Chow [3] had proved that the only compact complex submanifolds of complex projective space are those defined by a finite number of polynomial equations; thus the image of Kodaira's embedding map is always perforce an algebraic variety! Thus in many contexts it can be deduced that a given compact complex manifold-globally defined in some transcendental terms, for instance by a collection of holomorphic transition functions - is actually something completely describable in terms of elementary algebra, and algebraic geometric methods can then be brought to bear on further understanding the given space, often with stunning consequences. One might hope that, wherever this remarkable reduction from analysis to algebra is echoed in physics, there will be a strong tendency for the corresponding theory to be both better behaved and more full analyzable than one would otherwise be wont to expect.

In this paper we will discuss the projective embedding problem for complex supermanifolds. We begin, in Sect. 2, by giving a characterization, in terms of positive rank one locally free sheaves, of those complex supermanifolds admitting superholomorphic embeddings into complex superprojective space. In Sect. 3, this is translated into an obstruction theory involving classical cohomological invariants, which are then computed for some concrete examples. In Sect. 4, we give a supermanifold generalization of Chow's theorem and an analogue of the Segre embedding theorem. Finally, in Sect. 5, we prove a projective embedding theorem for families. In particular, we show that a family of complex supermanifolds of dimension $1 \mid \mathrm{m}$ is necessarily a family of projective algebraic varieties. From the standpoint of physical applications, the last is perhaps the most important result proved in this article, since it applies, in particular, to families of super Riemann surfaces. 


\section{The Kodaira Embedding Theorem for Supermanifolds}

We shall use Manin's book [17] as a general reference for the theory of supermanifolds, and we shall summarize the most important definitions here in order to establish terminology and notation. A complex supermanifold is a ringed space $(M, \mathscr{A})$, where $M$ is a topological space, and $\mathscr{A}$ is a sheaf of supercommutative rings on $M$ such that, if we let $\mathcal{N}$ be the ideal of nilpotents in $\mathscr{A}$, the following conditions are satisfied:

1. $(M, \mathcal{O})$ is a complex manifold, where $\mathcal{O}:=\mathscr{A} / \mathscr{N}$;

2. $\mathscr{E}:=\mathscr{N} / \mathscr{N}^{2}$ is locally free over $\mathcal{O}$;

3. $\mathscr{A}$ is locally isomorphic to the $\mathbb{Z}_{2}$-graded exterior algebra $\Lambda_{\mathscr{O}}^{*} \mathscr{E}$.

We denote the complex supermanifold $(M, \mathscr{A})$ simply by $M$ when there is no confusion with the underlying topological space-just as one does in classical complex manifold theory. Similarly, the sheaf $\mathscr{A}$ is called the structure sheaf of the complex supermanifold $M$, and will be denoted, when necessary, by $\mathscr{A}_{M}$. The complex manifold underlying the supermanifold $M$ is its so-called reduction, defined as $(M, \mathcal{O})$, where $\mathcal{O}=\mathscr{A} / \mathcal{N}$, and denoted by $M_{\text {rd }}$. The next classical object that naturally arises in the theory of complex supermanifolds is a holomorphic vector bundle over $M_{\text {rd }}$ whose sheaf of sections is the locally free sheaf $\mathscr{E}:=\mathscr{N} / \mathscr{N}^{2}$ of $\mathcal{O}$-modules. We call $\mathscr{E}$ the characteristic sheaf of the complex supermanifold $M$. A split complex supermanifold $(M, \mathscr{A})$ is defined by the property that $\mathscr{A} \cong \Lambda^{\bullet} \mathscr{E}$, i.e. the supercommutative sheaf of rings is simply given by an exterior algebra $\left(\mathbb{Z}_{2}\right.$-graded and supercommutative in the obvious manner) over a locally free sheaf on the underlying complex manifold; the latter sheaf then becomes the characteristic sheaf of the split supermanifold. Note that not every complex supermanifold is split (cf. $[5,17,22]$ ). A family of simple but important examples of complex supermanifolds is given by the superprojective spaces.

Definition 1. Complex superprojective $n \mid m$-space is the supermanifold

$$
\mathbb{P}_{n \mid m}=\left(\mathbb{P}_{n}, \Lambda^{\cdot}\left(\mathbb{C}^{m} \otimes_{\mathbb{C}} \mathcal{O}(-1)\right)\right),
$$

where $\mathcal{O}(-1)$ is the sheaf of sections of the tautological $\left(c_{1}=-1\right)$ line bundle over complex projective space $\mathbb{P}_{n}$.

The motivation for this definition is as follows: a "holomorphic function" on $\mathbb{P}_{n \mid m}$ should surely be a function of total homogeneity 0 in $n+1$ even variables $\left(z^{0}, \ldots, z^{n}\right)$ and $m$ odd variables $\left(\theta^{1}, \ldots, \theta^{m}\right)$. Such a function could be uniquely represented as

$$
\sum_{k=0}^{m} \sum_{1 \leqq i_{1}<\cdots<i_{k} \leqq m} f_{i_{1} \cdots i_{k}}\left(z^{0}, \ldots, z^{n}\right) \theta^{i_{1}} \cdots \theta^{i_{k}},
$$

where $f_{i_{1} \cdots i_{k}}$ has homogeneity $-k$. But this is precisely what is meant by a section of the Grassmann bundle $\Lambda^{\bullet}\left(\mathbb{C}^{m} \otimes \mathcal{O}(-1)\right)$.

Notice that $\mathbb{P}_{n \mid m}$ is a split complex supermanifold. The characteristic sheaf of $\mathbb{P}_{n \mid m}$ is, of course, $\mathbb{C}^{m} \otimes \mathcal{O}(-1)$.

Let us also recall that a (superholomorphic) mapping

$$
f:\left(M_{1}, \mathscr{A}_{1}\right) \rightarrow\left(M_{2}, \mathscr{A}_{2}\right)
$$


between complex supermanifold is a pair consisting of a holomorphic mapping

$$
f_{\mathrm{rd}}:\left(M_{1}, \mathcal{O}_{1}\right) \rightarrow\left(M_{2}, \mathcal{O}_{2}\right)
$$

and a pull-back morphism

$$
f^{*}: f_{\text {rd }}^{-1} \mathscr{A}_{2} \rightarrow \mathscr{A}_{1}
$$

of sheaves of $\mathbb{Z}_{2}$-graded rings extending the pull-back morphism for holomorphic functions. Such a map is called an embedding if, in addition:

1. $f_{\mathrm{rd}}$ is an embedding, and

2. $f^{*}$ is a sheaf epimorphism.

In the presence of 1,2 is equivalent to:

3. the induced vector bundle morphism

$$
f_{\mathrm{rd}}^{*}: E_{2} \rightarrow E_{1},
$$

is surjective, where $E_{i} \rightarrow M_{i}$ is the holomorphic characteristic vector bundle of $M_{i}$ defined by

$$
\mathcal{O}\left(E_{i}\right):=\mathscr{E}_{i}:=\mathcal{N}_{i} / \mathcal{N}_{i}^{2}, \quad i=1,2
$$

This latter formulation is easier to verify in practice, and will be the one we will really use.

Let us now recall the statement of the usual Kodaira embedding theorem $[13,24]$; this states that a compact complex manifold $M$ can be holomorphically embedded in some complex projective space if and only if it admits a positive holomorphic line bundle $L \rightarrow M$. Here a holomorphic line bundle is called positive if and only if it has a Hermitian structure for which the curvature is a Kähler form; for $M$ a Riemann surface, this amounts to requiring that $\int_{M} c_{1}(L)>0$. A locally free rank one sheaf $\mathscr{L}$ is of the form $\mathcal{O}(L)$, where $L$ is a holomorphic line bindle. We say that such an $\mathscr{L}$ is positive if $L$ is a positive line bundle. The correct analog of this notion in the $\mathbb{Z}_{2}$-graded case is as follows.

Definition 2. A locally free rank one sheaf of $\mathscr{A}$-modules on a supermanifold $(M, \mathscr{A})$ is said to be positive if its restriction $\mathscr{L}_{\mathrm{rd}}$ to the underlying complex manifold is a positive rank one sheaf.

Here, the restriction of a sheaf $\mathscr{F}$ of $\mathscr{A}$-modules from the supermanifold $(M, \mathscr{A})$ to the underlying complex manifold $(M, \mathcal{O})$ means the sheaf $\mathscr{F}_{\text {rd }}:=\mathscr{F} / \mathscr{N} \mathscr{F}$ of 0 -modules.

Using the definition we have the following result.

Theorem 1. A complex supermanifold $M$ with compact reduction can be embedded in some superprojective space $\mathbb{P}_{n \mid m}$ if and only if it admits a positive rank one sheaf of $\mathscr{A}_{M^{-} \text {-modules. }}$

Proof. One direction is trivial. If $f: M \hookrightarrow \mathbb{P}_{n \mid m}$ is an embedding, then

$$
\mathscr{L}=f^{*}\left(\mathcal{O}(1) \otimes_{\mathcal{O}} \Lambda^{*}\left(\mathbb{C}^{m} \otimes \mathcal{O}(-1)\right)\right)
$$

is a positive rank one sheaf on $M$. The converse will be proved by showing that some power of any positive line bundle is precisely of this form for some $f$. 
Thus, we now assume that there exists a positive rank one sheaf $\mathscr{L}_{0}$ on $M_{\text {rd }}$ which extends as a rank one locally free sheaf $\mathscr{L}$ of $\mathscr{A}$-modules. Let $L \rightarrow M_{\text {rd }}$ be the positive line bundle defined by $\mathscr{L}_{0}=\mathcal{O}(L)$, and let $E \rightarrow M$ be the characteristic vector bundle defined by $\mathcal{O}(E)=\mathscr{E}$. For $k \in \mathbb{N}$, let $\mathscr{L}^{k}:=\mathscr{L} \otimes_{\mathscr{A}} \cdots \otimes_{\mathscr{A}} \mathscr{L}$ be the $k$-fold tensor product of $\mathscr{L}$ with itself, and let $\mathscr{L}_{(l)}^{k}:=\mathscr{L}^{k} / \mathscr{N}^{l+1} \mathscr{L}^{k}$. Thus

$$
\begin{aligned}
& \mathscr{L}_{(0)}^{k}=\mathcal{O}\left(L^{\otimes k}\right), \\
& \mathscr{L}_{(1)}^{k}=\mathscr{L}_{(0)}^{k} \otimes(\mathcal{O} \oplus \mathscr{E}), \\
& \mathscr{L}_{(j)}^{k}=\mathscr{L}^{k}, \quad j \geqq \operatorname{rank} E .
\end{aligned}
$$

More generally, we have the exact sequence

$$
0 \rightarrow \mathscr{L}_{(0)}^{k} \otimes \Lambda^{l} \mathscr{E} \rightarrow \mathscr{L}_{(l)}^{k} \rightarrow \mathscr{L}_{(l-1)}^{k} \rightarrow 0 .
$$

Lemma 1. The restriction map $\Gamma\left(M, \mathscr{L}^{k}\right) \rightarrow \Gamma\left(M, \mathscr{L}_{(1)}^{k}\right)$ is surjective if $k$ is sufficiently large.

Proof. From 1 we have exact sequences

$$
H^{0}\left(M, \mathscr{L}_{(l)}^{k}\right) \rightarrow H^{0}\left(M, \mathscr{L}_{(l-1)}^{k}\right) \rightarrow H^{1}\left(M, \mathscr{L}_{0}^{k} \otimes \Lambda^{l} \mathscr{E}\right)
$$

But by Grauert's vanishing theorem [8], the positivity of $L$ implies that $H^{1}\left(M, \mathscr{L}_{0}^{k} \otimes \Lambda^{l} \mathscr{E}\right)=0$ for any fixed $l$, provided that $k$ is sufficiently large. For $k$ sufficiently large we therefore have

$$
H^{1}\left(M, \mathscr{L}_{0}^{k} \otimes \Lambda^{l} \mathscr{E}\right)=0, \quad \text { for } \quad l=1,2, \ldots, \operatorname{rank} E .
$$

It then follows by induction that $\Gamma\left(M, \mathscr{L}^{k}\right) \rightarrow \Gamma\left(M, \mathscr{L}_{(1)}^{k}\right)$ is onto.

Let $\mathscr{I}_{x} \subset \mathcal{O}$ denote the ideal sheaf of $x \in M$. Then $\mathscr{I}$ is a coherent analytic sheaf, and we can again conclude by the Grauert vanishing theorem that, for $k$ sufficiently large,

and

$$
H^{1}\left(M, \mathscr{I}_{x} \otimes \mathscr{I}_{y} \otimes \mathscr{L}_{0}^{k}\right)=0
$$

$$
H^{1}\left(M, \mathscr{I}_{x} \otimes \mathscr{E} \otimes \mathscr{L}_{0}^{k}\right)=0
$$

for all $x, y \in M$. But we also have the short exact sequences (where $J^{1} F$ is the vector bundle of 1-jets of any vector bundle $F$ )

$$
\begin{aligned}
& 0 \rightarrow \mathscr{I}_{x} \otimes \mathscr{I}_{y} \otimes \mathscr{L}^{k} \rightarrow \mathscr{L}^{k} \rightarrow L_{x}^{k} \oplus L_{y}^{k} \rightarrow 0, \\
& 0 \rightarrow \mathscr{I}_{x}^{2} \otimes \mathscr{L}^{k} \rightarrow \mathscr{L}^{k} \rightarrow\left(J^{1} L^{k}\right)_{x} \rightarrow 0, \\
& 0 \rightarrow \mathscr{I}_{x} \otimes \mathscr{E} \otimes \mathscr{L}^{k} \rightarrow \mathscr{E} \otimes L^{k} \rightarrow E_{x} \otimes L_{x}^{k} \rightarrow 0,
\end{aligned}
$$

so the evaluation maps

$$
\begin{aligned}
\Gamma\left(M, \mathscr{L}^{k}\right) & \rightarrow L_{x}^{k} \oplus L_{y}^{k}, \\
\Gamma\left(M, \mathscr{L}^{k}\right) & \rightarrow\left(J^{1} L^{k}\right)_{x}, \\
\Gamma\left(M, \mathscr{E} \otimes L^{k}\right) & \rightarrow E_{x} \otimes L_{x}^{k},
\end{aligned}
$$

are all onto. Let $s_{0}, s_{1}, \ldots, s_{n}$ be a basis for $\Gamma\left(M, \mathscr{L}^{k}\right)$, and let $t_{1}, \ldots, t_{m}$ be a basis for $\Gamma\left(M, \mathscr{E} \otimes L^{k}\right)$. By Lemma 1 , these may be respectively extended as even sections $\sigma_{0}, \sigma_{1}, \ldots, \sigma_{n} \in \Gamma\left(M, \Lambda_{\text {even }}^{k}\right)$ and odd sections $\tau_{1}, \ldots, \tau_{m} \in \Gamma\left(M, \mathscr{L}_{\text {odd }}^{k}\right)$ of $\mathscr{L}^{k}$ 
To obtain our embedding, let us first define $f_{\mathrm{rd}}: M \rightarrow \mathbb{P}_{n}$ by

$$
f_{\mathrm{rd}}(x)=\left[\left\langle s_{0}(x), e_{x}\right\rangle,\left\langle s_{1}(x), e_{x}\right\rangle, \ldots,\left\langle s_{n}(x), e_{x}\right\rangle\right],
$$

where $e_{x}$ is any basis for $\left(L_{x}^{k}\right)^{*} \cong \mathbb{C}$. The surjectivity (2) guarantees that at least one component of our expression for $f_{\text {rd }}(x)$ is non-zero, so that $f_{\text {rd }}$ is a well-defined holomorphic map. The surjectivity (3) also says that $e_{x}$ and $e_{y}$ define linearly independent functionals $\Gamma\left(M, \mathscr{A}\left(L^{k}\right)\right) \rightarrow \mathbb{C}$, so that $f_{\mathrm{rd}}: M \rightarrow \mathbb{P}_{n}=\mathbb{P}\left(\Gamma\left(M, \mathcal{O}\left(L^{k}\right)\right)^{*}\right)$ is injective. Finally, the surjectivity (4) guarantees that $f_{\mathrm{rd}}$ is an immersion.

To extend this (usual Kodaira embedding) map to a map of supermanifolds, we simply need to specify $f^{*}$ on a set of generators. We may do this by defining

and

$$
f^{*}\left(\frac{z^{j}}{z^{k}}\right)=\frac{\sigma_{i}}{\sigma_{k}}
$$

$$
f^{*}\left(\frac{\theta^{j}}{z^{k}}\right)=\frac{\tau_{i}}{\sigma_{k}},
$$

where $z^{0}, \ldots, z^{n}$ and $\theta^{1}, \ldots, \theta^{m}$ are the usual homogeneous coordinates on $\mathbb{P}_{n \mid m}$, that is to say the standard basis for $\Gamma\left(\mathbb{P}_{n}, \mathcal{O}(1) \otimes_{\mathcal{O}} \Lambda^{*}\left(\mathbb{C}^{m} \otimes \mathcal{O}(-1)\right)\right)$.

To conclude, we need to check that $f^{*}$ is an epimorphism, or, equivalently, that the induced map

$$
f^{*}: \mathbb{C}^{m} \otimes \mathcal{O}(-1) \rightarrow \mathcal{O}(E)
$$

is surjective. But this is precisely the surjectivity assertion (4).

Corollary 2. Any split complex supermanifold $M$ whose reduction $M_{\mathrm{rd}}$ is projective algebraic is superprojective.

Proof. A split supermanifold $M$ is of the form $(M, \Lambda \mathscr{E})$ where $\mathscr{E}$ is the characteristic sheaf. Since $\mathcal{O}$ injectively embeds in $\mathscr{A}_{M}=\Lambda \mathscr{E}$ we have a natural projection $\pi: M \rightarrow M_{\mathrm{rd}}$. Thus, if $\mathscr{L}$ is a positive rank one locally free sheaf on $M_{\mathrm{rd}}$, then $\pi^{*} \mathscr{L}$ is a locally free sheaf of rank one on $M$ with the property that $\left(\pi^{*} \mathscr{L}\right)_{\mathrm{rd}}=\mathscr{L}$, and hence is positive on $M$. By Theorem 1, $M$ is superprojective.

We shall see less trivial applications in later sections.

We note at this point that the embedding provided by the proof of the theorem is not typically optimal, in the sense that if a complex supermanifold of dimension $r \mid s$ admits an embedding into some $\mathbb{P}_{n \mid m}$, it always embeds in $\mathbb{P}_{2 r+1 \mid r+s}$. One can do this by dropping a certain number of odd and even coordinates after a linear transformation. To see, for instance, that one can always eliminate an odd variable if $m>r+s$, notice that the pull-back map $\mathbb{C}^{m} \otimes \mathcal{O}(-1) \rightarrow \mathscr{E}$ induced by an embedding gives rise to a map $E^{*}(-1) \rightarrow \mathbb{C}^{m}$ whose image is a complex cone. The total space of $E^{*}(-1) \rightarrow M$ has complex dimension $r+s$, so if $r+s<m$ the image of $E^{*}(-1) \rightarrow \mathbb{C}^{m}$ has measure 0 , and consequently there exists a one-dimensional subspace $\mathbb{C} \subset \mathbb{C}^{m}$ which meets the image of $E^{*}(-1)$ only at 0 ; projecting to a complementary $\mathbb{C}^{m-1}$ then gives rise to a map $(M, \mathscr{A}) \rightarrow \mathbb{P}_{n \mid m-1}$, which is, by construction, an embedding. Reducing $n$ to $2 r+1$ is similar and completely standard. 
We close this section by mentioning a result of Penkov [19] which gives a necessary and sufficient condition that a complex super Grassmannian is embeddable in complex superprojective space. Following Manin [17], we let $G(r|s ; n+r| m+s)$ denote the super Grassmannian manifold of free regular submodules of $\mathbb{C}^{n+r \mid m+s}$ of rank $r \mid s$. This set can be endowed with a topology and with a structure sheaf to make it a complex supermanifold of dimension $r n \mid s m$. In general a super Grassmannian is not a split complex supermanifold [17], and ınore generally, Penkov shows that the super Grassmannian manifold $G(r|s ; n+r| m+s)$ can be embedded in superprojective space if and only if $r=0$ or $s=0$ or $n=0$ or $m=0$. In particular the simplest example of such a nonembeddable manifold is of the form $G(1|1 ; 2| 2)$. As we see above (2), all split supermanifolds admit projective embeddings, and thus for example $G(1|1 ; 2| 2)$ is not split. Nonetheless, as we shall see in the next section, many interesting non-split supermanifolds do embed in super projective space.

\section{Classical Invariants and the Embedding Problem}

According to Theorem 1, a supermanifold is projective iff there is a line bundle on the supermanifold $M$ such that its restriction to the reduced space is a positive line bundle in the classical sense, i.e. iff there is a positive invertible $\mathcal{O}$-module which can be extended to be an invertible $\mathscr{A}_{M}$-module on $M$. In this section, we will therefore be able to describe the obstruction to embedding $M$ in superprojective space in two different, albeit equivalent, ways.

Suppose that $M$ is a supermanifold with projective algebraic reduced space $M_{\text {rd }}$ and characteristic vector bundle $E \rightarrow M_{\text {rd }}$. Thus, there is, by assumption, a very ample invertible sheaf $\mathscr{L}_{(0)}$ of $\mathcal{O}$-modules on $M_{\text {rd }}$. In fact, by passing to a tensor power of a given positive line bundle, we can take $\mathscr{L}_{(0)}$ to be sufficiently ample so that $\mathscr{L}_{(0)}^{n} \otimes \Lambda^{k} \mathscr{E}$ is generated by its global sections for all $n \geqq 1, k \geqq 0$ and $H^{r}\left(M, \mathscr{L}_{(0)}^{n} \otimes \Lambda^{k} \mathscr{E}\right)=0$ for all $n \geqq 1, k \geqq 0$, and $r \geqq 1$. Let $\phi_{0}: M_{\mathrm{rd}} \rightarrow \mathbb{P}_{n}$ be the embedding defined by the sections of $\mathscr{L}_{(0)}$; we will call such an embedding very ample. Then $\phi_{0}^{*} \mathcal{O}(1)=\mathscr{L}_{(0)}$, where we let $\mathcal{O}(1)$ be the sheaf of holomorphic sections of the hyperplane line bundle on $\mathbb{P}_{n}$ (so that $\left.c_{1}(\mathcal{O}(1))=1\right)$. As $\mathscr{L}_{(0)} \otimes \mathscr{E}$ is generated by global sections, one has a surjection:

$$
\left.\mathscr{F}\right|_{M} \rightarrow \mathscr{E} \rightarrow 0,
$$

where $\mathscr{F}=\oplus^{m} \mathcal{O}(-1)$, because $\left.\mathcal{O}(-1)\right|_{M}=\phi_{0}^{*} \mathcal{O}(-1)=\mathscr{L}_{(0)}^{-1}$.

Theorem 3. Let $(M, \mathscr{A})$ be a complex supermanifold with compact reduction $M_{\mathrm{rd}}$, and let $\phi_{0}: M_{\mathrm{rd}} \subseteq \mathbb{P}_{n}$ be a very ample projective embedding. Then the obstructions to extending $\phi_{0}$ to an embedding $\phi: M \hookrightarrow \mathbb{P}_{n \mid m}$ are elements of $H^{2}\left(M_{\mathrm{rd}}, \Lambda^{2 k} \mathscr{E}\right)$, for $k=1,2, \ldots,[\operatorname{rank} E / 2]$.

First Proof. Up to isomorphism, a line bundle on $(M, \mathscr{A})$ is given by an element of $H^{1}\left(M, \mathscr{A}_{* \text { even }}\right)$, where $\mathscr{A}_{* \text { even }}$ is the multiplicative sheaf of even invertibles. Let $\mathscr{S}_{2 k}$ be the normal subgroup of elements of the form $1+\alpha$, where $\alpha \in \mathscr{N}^{2 k}$; we define $\mathscr{A}_{* 2 k}=\mathscr{A}_{* \text { even }} / \mathscr{S}_{2(k+1)}$. Then $\mathscr{A}_{* 0}=\mathcal{O}_{*}$, the sheaf of non-zero holomorphic functions, and $\mathscr{A}_{* 2 k}=\mathscr{A}_{* \text { even }}$ if $2 k>q$. We have exact sequences 
$0 \rightarrow \Lambda^{2 k} \mathscr{E} \rightarrow \mathscr{A}_{* 2 k} \rightarrow \mathscr{A}_{* 2(k-1)} \rightarrow 0$ so an element of $H^{1}\left(M_{1}, \mathcal{O}_{*}\right)$ extends to an element of $H^{1}\left(M, \mathscr{A}_{* \text { even }}\right)$ if and only if a sequence of obstructions vanish, where these obstructions are elements of $H^{2}\left(M_{\mathrm{rd}}, \Lambda^{2 k} \mathscr{E}\right)$.

Second Proof. Let $M^{(k)}=\left(M, \mathscr{A} / \mathscr{N}^{k}\right)$ be the $k^{\text {th }}$ neighborhood of $M_{\text {rd }}$ in $M$. One then has a natural sequence of embeddings of ringed spaces:

$$
M_{\mathrm{rd}}=M^{(0)} \hookrightarrow M^{(1)} \hookrightarrow \cdots \hookrightarrow M^{(q-1)} \sqsubset M^{(q)}=M .
$$

We extend the embedding

to

$$
\phi^{(0)}: M^{(0)} \stackrel{\phi_{0}}{\longrightarrow} \mathbb{P}_{n} \stackrel{{ }^{i}}{\longrightarrow} \mathbb{P}_{n \mid m}
$$

$$
\phi^{(l)}: M^{(l)} \subseteq \mathbb{P}_{n \mid m}
$$

by induction. Thus, assume that we have an embedding for $l=k-1$. Locally, there is no obstruction to extending this map to a map for $l=k$, but there are many ways of making such an extension, the choices being parametrized by $\left(\phi^{*} \mathscr{D} \otimes \Lambda^{k} \mathscr{E}\right)_{\text {even }}$, where $\mathscr{D}$ denotes the sheaf of derivations of the sheaf of algebras $\left.\Lambda \mathscr{F}\right|_{M}$ on $\mathbb{P}_{n}$. Hence the obstruction to making a consistent global choice is an element of $H^{1}\left(M_{\text {rd }},\left(\phi^{*} \mathscr{D} \otimes \Lambda^{k} \mathscr{E}\right)_{\text {even }}\right)$. On the other hand,

$$
\phi^{*} \mathscr{D} \cong \phi^{*} \mathscr{T} \oplus \phi^{*} \mathscr{F} *
$$

where $\mathscr{T}$ is the tangent sheaf of $\mathbb{P}_{n}$. Therefore, when $k$ is even, the obstruction is in $H^{1}\left(M_{\mathrm{rd}}, \phi^{*} \mathscr{T} \otimes \Lambda^{k} \mathscr{E}\right)$; and when $k$ is odd, the obstruction is in $H^{1}\left(M_{\mathrm{rd}}, \phi^{*} \mathscr{F}^{*} \otimes \Lambda^{k} \mathscr{E}\right)$.

However, $\phi^{*} \mathscr{F} *$ is a finite (direct) sum of the very ample invertible sheaf $\mathscr{L}_{(0)}$. By our choice of $\mathscr{L}_{(0)}$, the last cohomology group vanishes. Therefore, there is no obstruction when $k$ is odd.

On the other hand, one has the Euler sequence on $\mathbb{P}_{n}$ :

$$
0 \rightarrow \mathcal{O} \rightarrow \mathcal{O}(1)^{\otimes(n+1)} \rightarrow \mathscr{T} \rightarrow 0 .
$$

Pulled back by $\phi$ onto $M$ and then tensored by $\Lambda^{k} \mathscr{E}$, this yields

$$
0 \rightarrow \Lambda^{k} \mathscr{E} \rightarrow \Lambda^{k} \mathscr{E} \otimes \phi^{*} \mathcal{O}(1)^{\oplus(n+1)} \rightarrow \Lambda^{k} \mathscr{E} \otimes \phi^{*} \mathscr{T} \rightarrow 0,
$$

or, in other words,

$$
0 \rightarrow \Lambda^{k} \mathscr{E} \rightarrow \Lambda^{k} \mathscr{E} \otimes \mathscr{L}_{(0)}^{\oplus(n+1)} \rightarrow \Lambda^{k} \mathscr{E} \otimes \phi^{*} \mathscr{T} \rightarrow 0 .
$$

By our choice of $\mathscr{L}_{(0)}$, this exact sequence induces an isomorphism:

$$
H^{1}\left(M_{\mathrm{rd}}, \Lambda^{k} \mathscr{E} \otimes \phi^{*} \mathscr{T}\right) \cong H^{2}\left(M, \Lambda^{k} \mathscr{E}\right) .
$$

Therefore, the only obstruction of extension is in this second cohomology when $k$ is even.

\section{Remarks.}

- Classical analogues of the above proofs arise when one attempts to extend analytic objects off a complex submanifold $M_{\mathrm{rd}} \in M$ by power series. For example, the standard obstruction theory of Griffiths [10] then tells us that the obstructions 
to the extension of a map are in the first cohomology with values in the tensor product of the tangent sheaf and the symmetric powers of the conormal bundle $\mathscr{E}^{*}$, while the obstructions to extending a line bundle are in the second cohomology with values in the symmetric powers of the conormal bundle. In the category of supermanifolds, we have simply remarked that these principles still work, with the proviso that symmetric products are to be replaced with alternating ones. On the other hand, since the resulting obstructions now terminate after a finite number of steps, one is not confronted with the delicate convergence problems arising in the classical case.

- Notice that the first proof actually gives a criterion for extending any line bundle, not just a poisitive one.

- The obstruction theory of the second argument is sufficiently general to also, for example, show that one can always embed a supermanifold with Stein reduction in $\mathbb{C}^{n \mid m}$ as a closed submanifold; the generality of the method stems from the fact that it is more closely tied to the idea of a map. As another example of this same method in another setting, consider what happens if one replaces the superprojective space $\mathbb{P}_{n \mid m}$ by the space $M_{\text {rd }}$ and the map $\phi_{0}$ by the identity map; one rediscovers in this the obstructions to splitting the supermanifold $M$, which are elements in $H^{1}\left(M_{\mathrm{rd}}, \mathscr{T}_{\mathrm{rd}} \otimes \Lambda^{k} \mathscr{E}\right)$. The classical analogues are the obstructions to finding a holomorphic foliation transverse to a complex submanifold [10].

- These arguments also work in the $\mathscr{C}^{\infty}$ category, as all vanishing theorems can be replaced by the more elementary fact that $\mathscr{C}^{\infty}$ sheaves are fine. Of course, all smooth supermanifolds admit not only superprojective embeddings, but as previously noted, affine embeddings as well.

Corollary 4. Any supermanifold of dimension $1 \mid m$ is superprojective.

This follows from the fact that $H^{2}(M, \mathscr{F})=0$ for any coherent analytic sheaf $\mathscr{F}$ on any Riemann surface $M$ simply because $2>\operatorname{dim}_{\mathbb{C}} M$.; cf. [11]. By contrast, for the super Grassmannian $G(1|1 ; 2| 2)$ the obstructions are not trivial.

We close this section by giving some higher dimensional examples of complex supermanifolds which do not split, but which admit a superprojective embedding.

Example. Suppose that $\mathscr{E}$ is a rank 2 locally free sheaf of $\mathcal{O}$-modules with determinant bundle $\mathscr{L}_{(0)}$. Let $\left(M, \Lambda^{\circ} \mathscr{E}\right)$ be a split supermanifold. A theorem of Rothstein [22] states that, for any $k \geqq 2$, if $H^{2}\left(M, \operatorname{Der}^{(2 k)} \Lambda^{\circ} \mathscr{E}\right)=\{0\}$, then there is an analytic family of supermanifolds parametrized by $H^{1}\left(M, \operatorname{Der}^{(k)} \Lambda^{*} \mathscr{E} / \operatorname{Der}^{(2 k)} \Lambda^{0} \mathscr{E}\right)$, where

$$
\operatorname{Der}^{(j)} \Lambda^{\cdot} \mathscr{E} \equiv \sum_{j \leqq 2 k \leqq n} \operatorname{Der}_{2 k} \Lambda^{\cdot} \mathscr{E}
$$

and $Y$ is an element in $\operatorname{Der}_{l} \Lambda^{\bullet} \mathscr{E}$ if locally with coordinates $\left(z^{1}, \ldots, z^{m}, \theta^{1}, \ldots, \theta^{n}\right)$,

$$
Y=\sum f^{j} \partial_{z^{j}}+g^{k} \partial_{\theta^{k}},
$$

where $f^{j}$ and $g^{k}$ are $\mathcal{O}$-sections of $\Lambda^{\bullet} \mathscr{E}$ with degree $l$ and $l+1$ respectively.

Now, since $\mathscr{E}$ has rank 2 and the highest degree of $\Lambda^{\circ} \mathscr{E}$ is 2 ,

$$
\operatorname{Der}^{(4)} \Lambda^{\bullet} \mathscr{E}=0
$$


and

$$
\operatorname{Der}^{(2)} \Lambda^{\bullet} \mathscr{E}=\Lambda^{2} \mathscr{E} \otimes \mathscr{T},
$$

where $\mathscr{T}$ is the tangent sheaf of the manifold $(M, \mathcal{O})$.

Therefore, every class in $H^{1}\left(M, \mathscr{L}_{(0)} \otimes \mathscr{T}\right)$ determines an isomorphism class of supermanifolds with characteristic sheaf $\mathscr{E}$. An isomorphism class consists of split manifolds iff its orbit under the action of

$$
H^{0}\left(M, \Lambda^{2} \mathscr{E} \otimes \mathscr{T}\right)
$$

contains the origin. Thus, for example, there certainly would exist non-split deformations if,

and

$$
h^{1}\left(M_{\mathrm{rd}}, \Lambda^{2} \mathscr{E} \otimes \mathscr{T}\right) \neq 0
$$

$$
h^{0}\left(M_{\text {rd }}, \Lambda^{2} \mathscr{E} \otimes \mathscr{T}\right)=0 .
$$

Yet all such deformations would be superprojectivity embeddable if, at the same time,

$$
h^{2}\left(M_{\text {rd }}, \mathscr{L}_{(0)}\right)=0 \text {. }
$$

To find such example, let's take $M_{\text {rd }}=\mathbb{P}_{1} \times \mathbb{P}_{1}$. Let $\mathscr{L}_{(0)}=\mathcal{O}(p, q)$ be the line bundle of bidegree $(p, q)$, and assume that $p \geqq q$. Since $\mathscr{T} \cong \mathcal{O}(2,0) \oplus \mathcal{O}(0,2)$, the Künneth formula yields

$$
\begin{aligned}
h^{1}\left(M_{\mathrm{rd}}, \mathscr{T} \otimes \mathscr{L}_{(0)}\right) \\
=h^{1}\left(M_{\mathrm{rd}}, \mathcal{O}(2+p, q)\right)+h^{1}\left(M_{\mathrm{rd}}, \mathcal{O}(p, 2+q)\right) \\
=h^{0}\left(\mathbb{P}_{1}, \mathcal{O}(2+p)\right) h^{1}\left(\mathbb{P}_{1}, \mathcal{O}(q)\right)+h^{1}\left(\mathbb{P}_{1}, \mathcal{O}(2+p)\right) h^{0}\left(\mathbb{P}_{1}, \mathcal{O}(q)\right) \\
\quad+h^{0}\left(\mathbb{P}_{1}, \mathcal{O}(p)\right) h^{1}\left(\mathbb{P}_{1}, \mathcal{O}(2+q)\right)+h^{1}\left(\mathbb{P}_{1}, \mathcal{O}(p)\right) h^{0}\left(\mathbb{P}_{1}, \mathcal{O}(2+q)\right) \\
=h^{0}\left(\mathbb{P}_{1}, \mathcal{O}(2+p)\right) h^{0}\left(\mathbb{P}_{1}, \mathcal{O}(-2-q)\right)+h^{0}\left(\mathbb{P}_{1}, \mathcal{O}(-4-p)\right) h^{0}\left(\mathbb{P}_{1}, \mathcal{O}(q)\right) \\
\quad+h^{0}\left(\mathbb{P}_{1}, \mathcal{O}(p)\right) h^{0}\left(\mathbb{P}_{1}, \mathcal{O}(-4-q)\right)+h^{0}\left(\mathbb{P}_{1}, \mathcal{O}(-2-p)\right) h^{0}\left(\mathbb{P}_{1}, \mathcal{O}(2+q)\right) \\
\neq 0 \quad \text { if } \quad p \geqq-2 \geqq q .
\end{aligned}
$$

Meanwhile, if $p>-2 \geqq q$, Serre duality then implies that

$$
h^{2}\left(M_{\mathrm{rd}}, \mathscr{L}_{(0)}\right)=h^{0}\left(\mathbb{P}_{1} \times \mathbb{P}_{1}, \mathcal{O}(-2-p,-2-q)\right)=0 .
$$

Therefore, given the above constraint on the bidegree, one can deform the split manifold to a projective non-split manifold.

Example. In this paragraph, we shall study the borderline case of the last example. $M$ is again the $\mathbb{P}_{1} \times \mathbb{P}_{1}, \mathscr{E}$ is the sheaf $\mathcal{O}(-1,-1) \oplus \mathcal{O}(-1,-1)$. The space $M$ has a covering $\left\{U_{j}: j=1,2,3,4\right\}$ given by the product of the standard affine coordinates on the projective line. Let $\left(X_{j}, Y_{j}\right)$ be such coordinates on $U_{j}$; then

$$
\left(X_{1}, Y_{1}\right)=\left(X_{2}, Y_{2}^{-1}\right)=\left(X_{3}^{-1}, Y_{3}\right)=\left(X_{4}^{-1}, Y_{4}^{-1}\right) .
$$

Rothstein's deformation theory (cf. also [5]) then tells us that the deformation space of the split supermanifold $\left(\mathbb{P}_{1} \times \mathbb{P}_{1}, \Lambda^{\cdot} \mathscr{E}\right)$ is a two dimensional linear space. In fact, if one uses super coordinate $\left(x_{j}, y_{j}, \zeta_{j}, \eta_{j}\right)$ over $U_{j}$ such that $x_{j, \mathrm{rd}}=X_{j}, y_{j, \mathrm{rd}}=Y_{j}$, 
then a supermanifold can be defined by the following coordinate change:

$$
\begin{aligned}
\left(x_{1}, y_{1}, \zeta_{1}, \eta_{1}\right) & =\left(x_{2}-s y_{2}^{-1} \zeta_{2} \eta_{2}, y_{2}^{-1},-y_{2}^{-1} \zeta_{2}, y_{2}^{-1} \eta_{2}\right) \\
& =\left(x_{3}^{-1}, y_{3}+t x_{3}^{-1} \zeta_{3} \eta_{3}, x_{3}^{-1} \zeta_{3},-x_{3}^{-1} \eta_{3}\right) \\
& =\left(x_{4}^{-1}+s x_{4}^{-2} y_{4}^{-1} \zeta_{4} \eta_{4}, y_{4}^{-1}-t x_{4}^{-1} y_{4}^{-2} \zeta_{4} \eta_{4},-x_{4}^{-1} y_{4}^{-1} \zeta_{4},-x_{4}^{-1} y_{4}^{-1} \eta_{4}\right),
\end{aligned}
$$

for $(s, t) \in \mathbb{C}^{2}$. We shall denote by $M(s, t)$ the supermanifold defined by the deformation with parameter value $(s, t)$. Thus $M(0,0)$ is the split model, and $M(1,1)$ is the supergrassmannian $G(1|1,2| 2)$.

Proposition 5. The locally free sheaf $\mathcal{O}(p, q)$ on $\mathbb{P}_{1} \times \mathbb{P}_{1}$ can be extended to be a locally free sheaf on $M(s, t)$ if and only if $s p+t q=0$.

Note that this proposition gives us a complete picture of the Picard group of the supermanifold $M(s, t)$. As a consequence, one can easily find supermanifolds that are not split but embeddable, e.g. when $(s, t)=(1,-1)$, or that are not split and not embeddable, e.g. when $(s, t)=(1,1)$.

The proof of this proposition is simply a standard computation of Čech cohomology; cf. [17]. For example, one may begin with the extension defined by

$$
\begin{aligned}
& g_{12}\left(X_{1}, Y_{1}\right)=y_{1}^{q}, g_{13}\left(X_{1}, Y_{1}\right)=x_{1}^{p}, \\
& g_{14}\left(X_{1}, Y_{1}\right)=x_{1}^{p} y_{1}^{q}, g_{23}\left(X_{2}, Y_{2}\right)=x_{2}^{p} y_{2}^{q}, \\
& g_{24}\left(X_{2}, Y_{2}\right)=x_{2}^{p}, g_{34}\left(X_{3}, Y_{3}\right)=y_{3}^{q} .
\end{aligned}
$$

The obstruction to extension as a locally free sheaf is an element of $H^{2}\left(M_{\text {rd }}, \mathscr{T} \otimes \Lambda^{2} \mathscr{E}\right) \cong \mathbb{C}$ and is represented by the number $s p+t q$. In fact, when this obstruction is equal to zero, there is a unique way to write down the extension, namely,

$$
\begin{aligned}
& g_{12}\left(X_{1}, Y_{1}\right)=y_{1}^{q}, g_{13}\left(X_{1}, Y_{1}\right)=x_{1}^{p}, \\
& g_{14}\left(X_{1}, Y_{1}\right)=x_{1}^{p} y_{1}^{q}+t q x_{1}^{p-1} y_{1}^{q-1} \zeta_{1} \eta_{1}, \\
& g_{23}\left(X_{2}, Y_{2}\right)=x_{2}^{p} y_{2}^{q}+t q x_{2}^{p-1} y_{2}^{q-1} \zeta_{2} \eta_{2}, \\
& g_{24}\left(X_{2}, Y_{2}\right)=x_{2}^{p}, g_{34}\left(X_{3}, Y_{3}\right)=y_{3}^{q} .
\end{aligned}
$$

\section{Chow's Theorem and the Segre Embedding Theorem}

The complement to the classical Kodaira embedding theorem is the celebrated result of Chow which asserts that any embedded submanifold of projective space is defined by algebraic equations [3]. We now want to show that the image of a super projective embedding is a superalgebraic variety.

Let us begin by recalling that a homogeneous polynomial of degree $d$ in $n+1$ even variables $z^{0}, \ldots, z^{n}$ and in $m$ odd variables $\theta^{1}, \ldots, \theta^{m}$ is, by definition, an element of

$$
\bigoplus_{p+q=d}\left[\left(\odot^{p} \mathbb{C}^{n+1}\right) \otimes\left(\Lambda^{q} \mathbb{C}^{m}\right)\right]
$$

Thus, such a polynomial has a unique representation as

$$
p\left(z^{0}, \ldots, z^{n}, \theta^{1}, \ldots, \theta^{m}\right)=\sum_{q=0}^{d} \sum_{1 \leqq i_{1}<\cdots<i_{q} \leqq m} p_{\left\{i_{1}, \ldots, i_{q}\right\}}\left(z^{0}, \ldots, z^{n}\right) \theta^{i_{1}} \ldots \theta^{i_{q}},
$$


where $p_{\left\{i_{1}, \ldots, i_{q}\right\}}\left(z^{0}, \ldots, z^{n}\right)$ is a homogeneous polynomial of degree $d-q$ (in the usual sense); the polynomial $p_{\varnothing}\left(z^{0}, \ldots, z^{n}\right)$ corresponding to $q=0$ is, in particular, a homogeneous polynomial of degree $d$, and we will henceforth refer to it as the reduction $p_{\mathrm{rd}}$ of $p$.

Such a polynomial has another incarnation, which is central for our purposes. Consider the line bundle

$$
\mathscr{A}(d):=\mathcal{O}(d) \otimes_{\mathcal{O}} \Lambda^{\bullet}\left(\mathbb{C}^{m} \otimes \mathcal{O}(-1)\right)
$$

over $\mathbb{P}_{n \mid m}$. Then every global section of $\mathscr{A}(d)$ is given by a unique homogeneous polynomial $p\left(z^{0}, \ldots, z^{n}, \theta^{1}, \ldots, \theta^{m}\right)$ of degree $d$, and every such polynomial conversely defines a global section of $\mathscr{A}(d)$. The importance of this result stems from the fact that every line bundle over $\mathbb{P}_{n \mid m}, n \geqq 2$, is necessarily isomorphic to $\mathscr{A}(d)$ for some $d$.

Remark. By contrast, here are non-trivial continuous deformations of any line bundle over $\mathbb{P}_{1 \mid m}, m \geqq 2$. Indeed, as noted in the first proof of Theorem 3, rank one locally free sheaves of any supermanifold are classified by $H^{1}\left(\mathscr{A}_{* \text { even }}\right)$. As before, this may be analyzed via the exact sequences

$$
0 \rightarrow \Lambda^{2 k} \mathscr{E} \stackrel{\text { exp }}{\longrightarrow} \mathscr{A}_{* 2 k} \rightarrow \mathscr{A}_{* 2(k-1)} \rightarrow 1,
$$

where $\mathscr{A}_{* 2 k}=\mathscr{A}_{* \text { even }} / \exp \mathscr{N}^{2(k+1)}$ and $\mathscr{E}$ is the characteristic sheaf of $M:=\mathscr{N} / \mathscr{N}^{2}$. The claim follows from the fact that

$$
H^{1}\left(\mathbb{P}_{1}, \Lambda^{k}\left(\mathbb{C}^{m} \otimes \mathcal{O}(-1)\right)\right) \neq 0 \quad \text { if } \quad m \geqq k \geqq 2 .
$$

In a similar vein, using standard obstruction theory (cf. [5]), one may easily see that $\mathbb{P}_{n \mid m}$ is rigid for $n \geqq 2$, while $\mathbb{P}_{1 \mid m}$ has nontrivial deformations for $m \geqq 3$. These examples should serve as a warning to those who naively expect every result to remain true whenever the word "super" is inserted in all appropriate places. Nonetheless, enough does continue to be true so that this naive faith needn't be considered imbecilic.

Now suppose that we have a collection $p^{1}, \ldots, p^{N}$, depending on $n+1$ even variables $z^{0}, \ldots, z^{n}$ and in odd variables $\theta^{1}, \ldots, \theta^{m}$. Assume, moreover, that each of the given polynomials is of definite parity, meaning that, when represented as in (5), nonzero coefficients occur either only for $q$ even or only for $q$ odd. We can then produce an ideal $\mathscr{I}=\left\langle p^{1}, \ldots, p^{n}\right\rangle \subset \mathscr{A}=\mathscr{A}(0)$ by taking $\mathscr{I}$ to be generated, for $z^{j} \neq 0$, by $p^{k} /\left(z^{j}\right)^{d_{k}}$. Such an ideal will be called a superalgebraic ideal. Associated to this ideal is the graded ringed space $\left(X,\left.(\mathscr{A} / \mathscr{I})\right|_{X}\right)$, where $X \subset \mathbb{P}_{n}$ is the algebraic variety on which $\mathscr{A} / \mathscr{I}$ is supported. Such a graded ringed space will be called a complex superalgebraic variety.

Theorem 6. Let $(M, \mathscr{A})$ be a complex supermanifold such that the underlying complex manifold $M_{\mathrm{rd}}$ is compact. Suppose that $f:(M, \mathscr{A}) \rightarrow \mathbb{P}_{n \mid m}$ is an embedding. Then the image of $f$ is a complex superalgebraic variety. More precisely, the kernel of $f_{\mathrm{rd}}^{*}: f^{-1} \mathscr{A}_{\mathbb{P}_{n \mid m}} \rightarrow \mathscr{A}$ is of the form $f^{-1} \mathscr{I}$, where $\mathscr{I} \subset \mathscr{A}_{\mathbb{P}_{n \mid m}}$ is a superalgebraic ideal, and $f$ therefore induces an isomorphism between $(M, \mathscr{A})$ and the complex superalgebraic variety defined by $\mathscr{I}$.

Proof. Suppose that we are given an embedding $f:\left(M, \mathscr{A}_{M}\right) \subseteq \mathbb{P}_{n \mid m}$. In particular, 
we have an embedding $f_{\mathrm{rd}}: M \hookrightarrow \mathbb{P}_{n}$, and the classical Chow Theorem [3] says that there are a finite number of homogeneous polynomials $p_{\mathrm{rd}}^{1}, \ldots, p_{\mathrm{rd}}^{N}$ of $\left(z^{0}, \ldots, z^{n}\right)$, whose common zeros locus is $f_{\mathrm{rd}}(M)$ and which generate the ideal $\mathscr{J}$ of $f_{\mathrm{rd}}(M) \subset \mathbb{P}_{n}$. Notice, moreover, that we may choose the degrees of these polynomials to be larger than any given integer $l$ by replacing $p_{\mathrm{rd}}^{k}$ with $\left(z^{j}\right)^{l} p_{\mathrm{rd}}^{k}$ as necessary.

Now each of these polynomials defines a section of $\mathcal{O}\left(d_{j}\right) \subset \mathscr{A}\left(d_{j}\right)$ so that $f^{*}\left(p_{\mathrm{rd}}^{k}\right) \in \Gamma\left(M, \mathscr{L}_{j}^{d}\right)$ where $\mathscr{L}$ is the pull-back of $\mathscr{A}(1)$ to $M$. As was observed above, we may take $d_{k}=d$ for all $k$, where $d$ is as large as we like. Now we are faced with the problem of modifying $p_{\mathrm{rd}}^{k}$ by terms involving $\theta^{1}, \ldots, \theta^{m}$ so that the modified homogeneous polynomials $p^{k}$ satisfy $f^{*}\left(p^{k}\right)=0$ for all $k$. We do this by using the following result:

Lemma 2. Let $u \in \Gamma\left(M, \mathscr{N} \mathscr{L}_{(k)}^{\mathrm{d}}\right)$, where $\mathscr{N} \subset \mathscr{A}_{M}$ is the subsheaf of nilpotent elements, and where $\mathscr{L}_{(k)}^{d}:=\mathscr{L}^{d} / \mathscr{N}^{k+1} \mathscr{L}^{d}$. Then there is some $\hat{u} \in \Gamma\left(\mathbb{P}_{n}, \mathcal{O}(d) \otimes\right.$ $\left.\bigoplus_{q>0} \Lambda^{q}\left(\mathbb{C}^{m} \otimes \mathcal{O}(-1)\right)\right)$ whose restriction is $u$, at least for sufficiently large $d$. If $u$ has definite parity, $\hat{u}$ may also be chosen to have definite parity.

Proof. We do this by induction on $k$. If $k=1$, then $u$ is an element of $\Gamma\left(M, \mathcal{O}\left(L^{d} \otimes E\right)\right)$, and we may consider the pair of exact sequences,

$$
\begin{aligned}
& 0 \rightarrow \mathcal{O}_{M}\left(L^{d} \otimes F_{1}\right) \rightarrow \mathcal{O}_{M}\left(L^{d} \otimes\left(\mathbb{C}^{m} \otimes L^{-1}\right)\right) \rightarrow \mathcal{O}_{M}\left(L^{d} \otimes E\right) \rightarrow 0, \\
& 0 \rightarrow \mathscr{J} \otimes \mathcal{O}_{\mathbb{P}_{n}}(d) \otimes \mathbb{C}^{m} \otimes \mathcal{O}_{\mathbb{P}_{n}}(-1) \rightarrow \mathcal{O}_{\mathbb{P}_{n}}(d) \otimes \mathbb{C}^{m} \otimes \mathcal{O}(-1) \\
& \rightarrow \mathcal{O}_{M}\left(L^{d} \otimes\left(\mathbb{C}^{m} \otimes L^{-1}\right)\right) \rightarrow 0,
\end{aligned}
$$

concluding that, if $d$ is large enough so that, as predicted by Grauert's vanishing theorem, $H^{1}\left(M, \mathcal{O}\left(L^{d} \otimes F_{1}\right)\right)=H^{1}\left(\mathbb{P}_{n}, \mathscr{J} \otimes \mathbb{C}^{m} \otimes \mathcal{O}(d-1)\right)=0$, then there is an element

$$
\hat{u} \in \Gamma\left(\mathbb{P}_{n}, \mathcal{O}(d) \otimes \mathbb{C}^{m} \otimes \mathcal{O}(-1)\right) \subset \Gamma\left(\mathbb{P}_{n}, \mathcal{O}(d) \otimes \Lambda^{*}\left(\mathbb{C}^{m} \otimes \mathcal{O}(-1)\right)\right)
$$

whose restriction is $u$.

Now suppose that the statement holds for $k=1$, and that we are given an element $u \in \Gamma\left(M, \mathscr{N}_{\left.\mathscr{L}_{(k)}^{d}\right)}\right.$. By restricting $u$, we obtain an element $u_{k-1} \in \Gamma\left(M, \mathcal{N} \mathscr{L}_{(k-1)}^{d}\right)$ which, by hypothesis, extends as a "nilpotent" section $\hat{u}_{k-1}$ of $\mathscr{A}(d)$ on $\mathbb{P}_{n}$. The restriction of $\hat{u}_{k-1}$ to $\Gamma\left(M, \mathscr{N} \mathscr{L}_{(k)}^{d}\right)$ differs from $u$ by an element $v$ of $\Gamma\left(M, \mathcal{O}\left(L^{d} \otimes \Lambda^{k} E\right)\right)$. We have exact sequences

$$
0 \rightarrow \mathcal{O}_{M}\left(L^{d} \otimes F_{k}\right) \rightarrow \mathcal{O}_{M}\left(L^{d} \otimes \Lambda^{k}\left(\mathbb{C}^{m} \otimes L^{-1}\right)\right) \rightarrow \mathcal{O}_{M}\left(L^{d} \otimes \Lambda^{k} E\right) \rightarrow 0
$$

and,

$$
\begin{gathered}
0 \rightarrow \mathscr{J} \otimes \mathcal{O}_{\mathbb{P}_{n}}(d) \otimes \Lambda^{k}\left(\mathbb{C}^{m} \otimes \mathcal{O}_{\mathbb{P}_{n}}(-1)\right) \rightarrow \mathcal{O}_{\mathbb{P}_{n}}(d) \otimes \Lambda^{k}\left(\mathbb{C}^{m} \otimes \mathcal{O}_{\mathbb{P}_{n}}(-1)\right) \\
\rightarrow \mathcal{O}_{M}\left(L^{d} \otimes \Lambda^{k}\left(\mathbb{C}^{m} \otimes L^{-1}\right)\right) \rightarrow 0
\end{gathered}
$$

and, taking $d$ large enough so that

$$
H^{1}\left(M, \mathcal{O}\left(L^{d} \otimes F_{k}\right)\right)=0,
$$

and

$$
H^{1}\left(\mathbb{P}_{n}, \mathscr{J} \otimes \mathcal{O}(d-k) \otimes \Lambda^{k} \mathbb{C}^{m}\right)=0,
$$


we conclude that $v$ extends as an element

$$
\hat{v} \in H^{0}\left(\mathbb{P}_{n}, \mathcal{O}(d) \otimes \Lambda^{k}\left(\mathbb{C}^{m} \otimes \mathcal{O}(-1)\right)\right)=\Gamma\left(\mathbb{P}_{n}, \mathscr{A}(d)\right) .
$$

Then $\hat{u}=\hat{u}_{k-1}+\hat{v}$ is the desired extension.

To maintain the parity of $u$ when extending as $\hat{u}$, just consider either the odd or the even projection of $\hat{u}$, as appropriate.

To finish our proof of Theorem 6, we will need to apply Lemma 2 twice. The first application is to modify each $p_{\mathrm{rd}}^{k}$ so as to produce a new homogeneous polynomial with identical reduction but whose restriction to the embedded supermanifold $\left(M, \mathscr{A}_{M}\right)$ vanish. We may do this by letting

$$
r^{k} \in \Gamma\left(\mathbb{P}_{n}, \mathcal{O}(d) \otimes \underset{q>0}{\bigoplus_{>0}} \Lambda^{q}\left[\mathbb{C}^{m} \otimes \mathcal{O}(-1)\right]\right)
$$

be the constructed extension of $f^{*}\left(p_{\mathrm{rd}}^{k}\right)$, and letting $p^{k}:=p_{\mathrm{rd}}^{k}-r^{k}$. Notice that we may take the polynomials $p^{k}$ to all be even.

As our second application, let us first consider the exact sequence

$$
0 \rightarrow \mathscr{I}_{x}\left(E \otimes L^{d}\right) \rightarrow \mathcal{O}_{M}\left(E \otimes L^{d}\right) \rightarrow E_{x} \otimes L_{x}^{d} \rightarrow 0
$$

of sheaves on $M$, where $x \in M$ is any point, and where $\mathscr{I} \subset \mathcal{O}$ is the ideal sheaf of the point $x$. For $d$ sufficiently large, we have that

$$
H^{1}\left(M, \mathscr{I}_{x}\left(E \otimes L^{d}\right)\right)=0,
$$

so that elements of $\Gamma\left(M, \mathcal{O}\left(E \otimes L^{d}\right)\right)=\Gamma\left(M, \mathcal{N} \mathscr{L}_{(1)}^{d}\right)$ extend as odd homogeneous polynomials if $d$ is large. Thus we may find odd polynomials $p^{N+1}, \ldots, p^{N^{\prime}}$ in the ideal which generate the fibers of $E \otimes L^{d}$. In conjunction with $p^{1}, \ldots, p^{N}$, these polynomials constitute generators of the ideal, showing that $\mathscr{I}$ is superalgebraic. This then concludes the proof of Theorem 6 .

Example (Segre Embedding). Consider the super projective spaces $\mathbb{P}_{m \mid n}=\left(\mathbb{P}_{m}, \Lambda \mathscr{E}\right)$ and $\mathbb{P}_{k \mid l}=\left(\mathbb{P}_{k}, \Lambda \mathscr{F}\right)$, where $\mathscr{E}=\oplus^{n} \mathcal{O}(-1)$ and $\mathscr{F}=\oplus^{l} \mathcal{O}(-1)$. Their product space is the split manifold

$$
\mathbb{P}_{m \mid n} \times \mathbb{P}_{k \mid l}=\left(\mathbb{P}_{m} \times \mathbb{P}_{k}, \Lambda\left(\oplus^{n} \mathcal{O}(-1,0) \oplus^{l} \mathcal{O}(0,-1)\right),\right.
$$

where the bundle $\mathcal{O}(-1,0)$ or $\mathcal{O}(0,-1)$ is the pullback of a bundle from the first and second factor respectively. As it is a split manifold, according to Corollary 2, this supermanifold is superprojective and according to Theorem 6, it is superalgebraic. To find an embedding, let's follow the procedure in the proof of Theorem 3 and choose the $\mathcal{O}$-module $\mathcal{O}(1,0) \otimes \mathcal{O}(0,1)$ on the reduced space. This is a very ample invertible sheaf. Its associated map is exactly the Segre embedding from $\mathbb{P}_{m} \times \mathbb{P}_{k}$ into $\mathbb{P}_{m k+m+k+1}$. Since

$$
(\mathcal{O}(1,0) \otimes \mathcal{O}(0,1)) \otimes\left(\oplus^{n} \mathcal{O}(-1,0) \oplus^{l} \mathcal{O}(0,-1)\right)=\left(\oplus^{n} \mathcal{O}(0,1)\right) \oplus\left(\oplus^{l} \mathcal{O}(1,0)\right),
$$

this locally free sheaf is generated by global sections. In fact, one has

$$
\oplus^{n(k+1)+l(m+1)}(\mathcal{O}(-1,0) \otimes \mathcal{O}(0,-1)) \rightarrow \oplus^{n} \mathcal{O}(-1,0) \oplus^{l} \mathcal{O}(0,-1) \rightarrow 0 .
$$

Let $\Phi$ be the Segré embedding and $\mathcal{O}(1)$ the hyperplane bundle on $\mathbb{P}^{m k+m+k+1}$, the 
above exactly sequence can be expressed as

$$
\oplus^{n(k+1)+l(m+1)} \mathcal{O}(-1) \rightarrow \Phi_{*}\left(\oplus^{n} \mathcal{O}(-1,0) \oplus^{l} \mathcal{O}(0,-1)\right) \rightarrow 0 .
$$

In terms of supermanifolds, we have an embedding

$$
\mathbb{P}_{m \mid n} \times \mathbb{P}_{k \mid l} \rightarrow \mathbb{P}_{m k+m+k+1 \mid n(k+1)+l(m+1)} .
$$

To express the above map in coordinates, we take homogeneous coordinates $\left(z_{0}, z_{1}, \ldots, z_{m} ; \theta_{1}, \theta_{2}, \ldots, \theta_{n}\right)$ on $\mathbb{P}_{m \mid n}$ and $\left(w_{0}, w_{1}, \ldots, w_{k} ; \eta_{1}, \eta_{2}, \ldots, \eta_{l}\right)$ on $\mathbb{P}_{k \mid l}$. Then the "image" of the product in homogeneous coordinates, will be $\left(z_{i} w_{j}, w_{j} \theta_{\alpha}, \eta_{\beta} z_{i}\right)$ where $i=0,1, \ldots, m ; j=0,1, \ldots, k ; \alpha=1,2, \ldots, n ; \beta=1,2, \ldots, l$. Obviously, this is an embedding. However, this is not the Segre embedding which can be described algebraically as in the classical Segre embedding by the tensor product of submodules. In fact, given the "homogeneous" coordinates as above, they represent rank $(1 \mid 0)$ regular, i.e. nonnilpotent, submodules in $\mathbb{C}^{m+1 \mid n}$ and $\mathbb{C}^{k+1 \mid l}$ respectively. Their tensor product is a regular rank $(1 \mid 0)$ submodule in $\mathbb{C}^{(m+1)(k+1)+n l n(k+1)+l(m+1)}$. In coordinates, the Segré embedding sends the product to $\left(z_{i} w_{j},-\theta_{\alpha} \eta_{\beta} ; w_{j} \theta_{\alpha}, \eta_{\beta} z_{i}\right)$. Note that, at this level we are writing down the sheaf morphism and this is not a point set mapping. Let us study the products of $\mathbb{P}_{1 \mid 1}$ with itself. We have

$$
\Phi: \mathbb{P}_{1 \mid 1} \times \mathbb{P}_{1 \mid 1} \rightarrow \mathbb{P}_{4 \mid 4}
$$

given by coordinates as

$$
\left(z_{0}, z_{1} ; \theta\right) \times\left(w_{0}, w_{1} ; \eta\right) \rightarrow\left(z_{0} w_{0}, z_{0} w_{1}, z_{1} w_{0}, z_{1} w_{1},-\theta \eta ; z_{0} \eta, z_{1} \eta, \theta w_{0}, \theta w_{1}\right) .
$$

From this map one sees that the reduced map is from

$$
\mathbb{P}_{1} \times \mathbb{P}_{1} \rightarrow \mathbb{P}_{4}
$$

given by

$$
\left(z_{0}, z_{1}\right) \times\left(w_{0}, w_{1}\right) \rightarrow\left(z_{0} w_{0}, z_{0} w_{1}, z_{1} w_{0}, z_{1} w_{1}, 0\right),
$$

and when $\left(x_{0}, x_{1}, x_{2}, x_{3}, x_{4} ; \xi_{1}, \xi_{2}, \xi_{3}, \xi_{4}\right)$ represent coordinates on $\mathbb{P}_{4 \mid 4}$, the sheaf morphism $\phi^{*}$ is defined by

$$
\phi^{*} x_{0}=z_{0} w_{0}, \quad \phi^{*} x_{1}=z_{0} w_{1}, \quad \phi^{*} x_{2}=z_{1} w_{0}, \quad \phi^{*} x_{3}=z_{1} w_{1}, \quad \phi^{*} x_{4}=-\theta \eta ;
$$

and

$$
\phi^{*} \xi_{1}=z_{0} \eta, \quad \phi^{*} \xi_{2}=z_{1} \eta, \quad \phi^{*} \xi_{3}=\theta w_{0}, \quad \phi^{*} \xi_{4}=\theta w_{1} .
$$

As a superalgebraic variety, this product of superprojective spaces can be defined by the equations

$$
\begin{aligned}
& x_{0} x_{3}-x_{1} x_{2}=0, \quad x_{0} x_{4}-\xi_{1} \xi_{3}=0, \quad x_{1} x_{4}-\xi_{1} \xi_{4}=0, \quad x_{2} x_{4}-\xi_{2} \xi_{3}=0 \\
& x_{3} x_{4}-\xi_{2} \xi_{4}=0
\end{aligned}
$$

and

$$
x_{0} \xi_{2}-x_{2} \xi_{1}=0, \quad x_{0} \xi_{4}-x_{1} \xi_{3}=0, \quad x_{1} \xi_{2}-x_{2} \xi_{1}=0, \quad x_{2} \xi_{4}-x_{3} \xi_{3}=0 .
$$

Finally, the algebraic definition of Segre embedding can be generalized to show that the product of any super Grassmannians can be embedded into a super Grassmannian. As any flag manifold is submanifold of the product of Grassmannians, 
any flag manifold can be embedded into a grassmannian. In view of the fact that some super Grassmannian manifolds are not superprojective, one can raise the issue of embedding into super Grassmannians. In the category of manifolds and supermanifolds, one can simply say that a supermanifold can be embedded into a supergrassmannian if and only if there is a locally free sheaf of $\mathscr{A}$-module which is generated by global regular sections. Such a sheaf is certainly an extension of a locally free sheaf of $\mathcal{O}$-module which is generated by global sections. However, when we go through the same argument of the proof of Theorem 3, we find that the obstruction to the extension for an appropriately chosen sheaf of $\mathcal{O}$-modules from the reduced space to the ambient supermanifold consists also of elements in cohomology analogous to those described as in Theorem 3.

\section{A Superprojective Family Embedding Theorem}

We have seen that, for dimensional reasons, a supermanifold with dimension $1 \mathrm{|m}$ is always superprojective. However, it is more important to find out whether a family of such supermanifolds is necessarily projective in a suitable sense, since it is only in the context of families that the theory of super Riemann surfaces [6] becomes interesting.

Remark. A family of super Riemann surfaces is defined as a proper regular projection from a supermanifold $\left(X, \mathscr{A}_{X}\right)$ onto another supermanifold $\left(Y, \mathscr{A}_{Y}\right)$ such that the relative dimension is equal to $1 \mid 1$ and equipped with a rank $0 \mid 1$ locally free subsheaf $\mathscr{D}$ of the vertical tangent sheaf $\mathscr{T}_{X / Y}$ with the property that the map $[,]_{+}: \mathscr{D} \otimes \mathscr{D} \rightarrow \mathscr{T} / \mathscr{D}$ is an isomorphism

$$
\mathscr{D}^{2} \cong \mathscr{T}_{X / Y} / \mathscr{D}
$$

As a result, the reduced sheaf of $\mathscr{D}$ restricted to any fiber is simply a choice of the square root of the anticanonical bundle of the underlying Riemann surface. Let $\mathscr{L}$ be $\mathscr{D}^{-n}$ for some positive even integer, then $\mathscr{L}$ is a locally free rank one sheaf such that its restriction onto every fiber is positive.

This motivates the following definition:

Definition 3. Let $\left(X, \mathscr{A}_{X}\right) \stackrel{\pi}{\longrightarrow}\left(Y, \mathscr{A}_{Y}\right)$ be family of complex supermanifolds with compact reductions - i.e. we assume that $\pi_{\mathrm{rd}}$ is proper and that $\pi$ is submersive. $A$ relatively positive line bundle $\mathscr{L}$ on $\left(X, \mathscr{A}_{X}\right)$ is a locally free rank one sheaf of $\mathscr{A}_{X}$-modules whose restriction to each fiber $X_{y}:=\pi_{\mathrm{rd}}^{-1}(y), y \in Y$ is a positive line bundle.

We now establish a version of the direct image sheaf theorem of Grauert ([17]; cf. [14]).

Lemma 3. Let $\mathscr{L}$ be a relatively positive line bundle for the family $\left(X, \mathscr{A}_{X}\right) \stackrel{\pi}{\longrightarrow}\left(Y, \mathscr{A}_{Y}\right)$. For sufficiently large $l, H^{0}\left(X_{y}, \mathscr{L}_{y}^{l}\right)$ is locally independent of $y$.

More precisely, for any $y$, there is a neighborhood $U$ of $y$ and an integer $N$ such that for $l>N$ the natural restriction

$$
R_{y}^{0}: H^{0}\left(\pi^{-1}(U), \mathscr{L}^{l}\right) \rightarrow H^{0}\left(X_{y}, \mathscr{L}_{y}^{l}\right)
$$

is surjective for all $y \in U$. 
If, for such a neighborhood $U, \zeta$ is an element of $H^{0}\left(\pi^{-1}(U), \mathscr{L}^{l}\right)$ such that $R_{y}^{0} \zeta=0$ for all $y \in U$ then $\zeta=0$.

Proof. We have the exact sequence of sheaves of $\mathscr{A}_{X}$-modules:

$$
0 \rightarrow \mathscr{N}^{k+1} \rightarrow \mathscr{N}^{k} \rightarrow \Lambda^{k} \mathscr{E} \rightarrow 0 .
$$

Tensoring with $\mathscr{L}$, one has

$$
0 \rightarrow \mathcal{N}^{k+1} \mathscr{L} \rightarrow \mathscr{N}^{k} \mathscr{L} \rightarrow \Lambda^{k} \mathscr{E} \otimes \mathscr{L} \rightarrow 0 .
$$

Note that the $\mathscr{A}_{X}$-module $\Lambda^{k} \mathscr{E} \otimes \mathscr{L}$ is just the $\mathcal{O}_{X}$-module $\Lambda^{k} \mathscr{E} \otimes \mathscr{L}_{\text {rd }}$ and when $k=n$, the odd dimension of $\left(X, \mathscr{A}_{X}\right)$, then $\mathscr{N}^{n+1} \mathscr{L}=0$. Therefore, we have

$$
\begin{gathered}
0 \rightarrow \mathcal{N}^{k+1} \mathscr{L} \rightarrow \mathcal{N}^{k} \mathscr{L} \rightarrow \Lambda \mathscr{E} \otimes \mathscr{L}_{\text {rd }} \rightarrow 0, \quad 0 \leqq k \leqq n-1, \\
0 \rightarrow \mathscr{N}^{n} \mathscr{L} \rightarrow \Lambda^{n} \mathscr{E} \otimes \mathscr{L}_{\text {rd }} \rightarrow 0 .
\end{gathered}
$$

For any given $y$, choose an $l$ so large that for $j \geqq 1$ and for all $k$,

For each $k$, as the set

$$
H^{j}\left(X_{y}, \Lambda^{k} \mathscr{E}_{y} \otimes \mathscr{L}_{\mathrm{rd}}^{l}\right)=0 .
$$

$$
A_{j, 1}=\left\{y \in Y: \operatorname{dim} H^{j}\left(X_{y}, \Lambda^{k} \mathscr{E}_{y} \otimes\left(\mathscr{L}_{\mathrm{rd}}^{l}\right)_{y}\right) \geqq 1\right\}
$$

is an analytic subset $([17,14])$, one can choose a neighborhood $U$ of $y$ such that for all $y \in U$, and for all $k$,

$$
H^{j}\left(X_{y}, \Lambda^{k} \mathscr{E}_{y} \otimes\left(\mathscr{L}_{\mathrm{rd}}^{l}\right)_{y}\right)=0 .
$$

Then one can also deduce that $H^{0}\left(X_{y}, \Lambda^{k} \mathscr{E}_{y} \otimes\left(\mathscr{L}_{\text {rd }}^{l}\right)_{y}\right)$ is also independent of $y$ for all $y$ in $U$. By the Riemann-Roch formula on $X$, we know that, for sufficiently large $l$, such cohomology groups are independent of $y$ and that any higher cohomology are actually equal to zero. Restricting (6) and (7) onto any fiber $X_{y}$, we see from the induced cohomology exact seqence that the dimension of $H^{j}\left(X_{y}, \mathscr{L}_{y}^{l}\right)$ is independent of $y$.

- From now on, we shall simplify our notation by using $\mathscr{L}$ to denote our suitable positive line bundle $\mathscr{L}^{l}$.

As a consequence of the classical result of Kodaira and Grauert, the restriction map

$$
R_{y}^{j}: H^{j}\left(\pi^{-1}(U), \Lambda^{k} \mathscr{E} \otimes \mathscr{L}_{\mathrm{rd}}\right) \rightarrow H^{j}\left(X_{y}, \Lambda^{k} \mathscr{E}_{y} \otimes\left(\mathscr{L}_{\mathrm{rd}}\right)_{y}\right)
$$

is surjective and if $\zeta$ is an element in $H^{j}\left(\pi^{-1}(U), \Lambda^{k} \mathscr{E} \otimes \mathscr{L}_{\text {rd }}\right)$ such that $R_{y}^{j}(\zeta)=0$ for all $y \in U$, then $\zeta=0$. In particular, $H^{j}\left(\pi^{-1}(U), \Lambda^{k} \mathscr{E} \otimes \mathscr{L}_{\text {rd }}\right)=0$ for all $k$ and $j \geqq 1$. It follows that $H^{j}\left(\pi^{-1}(U), \Lambda^{k} \mathscr{E} \otimes \mathscr{L}\right)=0$. Then from (7), one can deduce that

$$
H^{j}\left(\pi^{-1}(U), \mathscr{N}^{n} \mathscr{L}\right)=0 \text { for all } j \geqq 1 .
$$

Taking the induced exact sequence of (6), we have

$$
H^{j}\left(\pi^{-1}(U), \mathscr{N}^{k} \mathscr{L}\right)=0 \text { for all } n \geqq k \geqq 0 .
$$

Then the induced long exact sequences of (6) and (7) over $\pi^{-1}(U)$ and over a fiber $X_{y}$ will yield the following exact sequences of cohomology:

$$
0 \rightarrow H^{0}\left(\pi^{-1} U, \mathscr{N}^{k+1} \mathscr{L}\right) \rightarrow H^{0}\left(\pi^{-1} U, \mathscr{N}^{k} \mathscr{L}\right) \rightarrow H^{0}\left(\pi^{-1} U, \Lambda^{k} \mathscr{E} \otimes \mathscr{L}\right) \rightarrow 0
$$


and

$$
0 \rightarrow H^{0}\left(X_{y},\left(\mathcal{N}^{k+1} \mathscr{L}\right)_{y}\right) \rightarrow H^{0}\left(X_{y},\left(\mathscr{N}^{k} \mathscr{L}\right)_{y}\right) \rightarrow H^{0}\left(X_{y},\left(\Lambda^{k} \mathscr{E} \otimes \mathscr{L}\right)_{y}\right) \rightarrow 0 .
$$

Moreover, the natural restrictions

and

$$
R_{y}^{k}: H^{0}\left(\pi^{-1}(U), \Lambda^{k} \mathscr{E} \otimes \mathscr{L}\right) \rightarrow H^{0}\left(X_{y},\left(\Lambda^{k} \mathscr{E} \otimes \mathscr{L}\right)_{y}\right) \text { for all } k
$$

are surjective.

$$
R_{y}^{n}: H^{0}\left(\pi^{-1}(U), \mathcal{N}^{n} \mathscr{L}\right) \rightarrow H^{0}\left(X_{y},\left(\mathcal{N}^{n} \mathscr{L}\right)_{y}\right)
$$

Again, induction will show that $R_{y}^{0}$ is surjective and that if $\zeta$ is an element in $H^{0}\left(\pi^{-1}(U), \mathscr{L}\right)$ such that $R_{y}^{0} \zeta=0$ for all $y \in U$, then $\zeta=0$. The proof of the lemma is completed.

As a consequence, we have the following ${ }^{1}$ :

Theorem 7. If $\left(X, \mathscr{A}_{X}\right) \rightarrow\left(Y, \mathscr{A}_{Y}\right)$ is a family of supermanifolds such that the space $\left(X, \mathscr{A}_{X}\right)$ has relatively positive line bundle, then for any point $y \in Y$, there is $a$ neighborhood $U$ of $y$ such that the family over the patch $U$ can be embedded into $\mathbb{P}_{n \mid m} \times\left(U, \mathscr{A}_{Y}(U)\right)$ such that the following diagram is commutative:

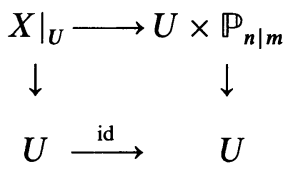

Proof. The lemma guarantees that, for a sufficiently positive power of the given line bundle, the embedding map from a fiber to $\mathbf{P}_{n \mid m}$ can be extended to a neighborhood in $X$. Taking the product with the projection to $U$ gives a map which is an embedding on some neighborhood.

Remark. If the base manifold is superprojective, it is now not difficult to use our generalization of Segre embedding to conclude that the total space of the family is also superprojective.

We now outline a version of the Chow theorem which is applicable in the present context of families. Suppose that we have a family

$$
\left(X, \mathscr{A}_{X}\right) \rightarrow\left(Y, \mathscr{A}_{Y}\right)
$$

which is embedded in $\left(Y, \mathscr{A}_{Y}\right) \times \mathbb{P}_{n \mid m}$ in a manner consistent with the projections so that the diagram

$$
\begin{array}{cc}
\left(X, \mathscr{A}_{X}\right) \stackrel{f}{\longrightarrow}\left(Y, \mathscr{A}_{Y}\right) \times \mathbb{P}_{n \mid m} \\
\downarrow \\
\downarrow \\
\left(Y, \mathscr{A}_{Y}\right) \stackrel{\text { id }}{\longrightarrow} & \left(Y, \mathscr{A}_{Y}\right)
\end{array}
$$

is commutative. We may then ask whether the image of $f$ may be defined by a finite number of homogeneous polynomials with coefficients which are elements

\footnotetext{
${ }^{1}$ In the case of super Riemann surfaces, Rabin and Topiwala [21], have independently formulated a similar result in a recent preprint
} 
of $\mathscr{A}_{Y}$, i.e., by functions of the form

$$
p(y, z, \theta)=\sum_{|I|+|J|=k} f_{I J}(y) z^{I} \theta^{J}
$$

where $(z, \theta)$ are homogeneous coordinates on $\mathbb{P}_{n \mid m}$, and $k$ is a positive integer. We shall see that this is locally true in $Y$.

Definition 4. Let $\left(X, \mathscr{A}_{X}\right)$ be a complex supersubmanifold of $\left(Y, \mathscr{A}_{Y}\right) \times \mathbb{P}_{n \mid m}$ such that the projection $\left(X, \mathscr{A}_{X}\right) \rightarrow\left(Y, \mathscr{A}_{Y}\right)$ is a family of complex supermanifolds with compact reduction. We say that $\left(X, \mathscr{A}_{X}\right)$ is projective superalgebraic over $\mathscr{A}_{Y}$ if $Y$ has an open covering $\left\{U_{\alpha}\right\}$ such that on each $U_{\alpha}$, there is a finite collection of superfunctions $\left\{g_{I J l \alpha}\right\}$ such that the ideal of $\left(X, \mathscr{A}_{X}\right) \subset\left(Y, \mathscr{A}_{Y}\right) \times \mathbb{P}_{n \mid m}$ is generated by the functions

$$
p_{l \alpha}=\sum_{I J} g_{I J l \alpha} z^{I} \theta^{J}
$$

where $|I|+|J|=$ constant for each $(l, \alpha)$.

Theorem 8 (Relative Chow Theorem). Let $\left(X, A_{X}\right) \rightarrow\left(Y, \mathscr{A}_{Y}\right)$ be a proper family of complex supermanifolds, and let $f:\left(X, \mathscr{A}_{X}\right) \rightarrow\left(Y, \mathscr{A}_{Y}\right) \times \mathbb{P}_{n \mid m}$ be an embedding of this family so that the diagram

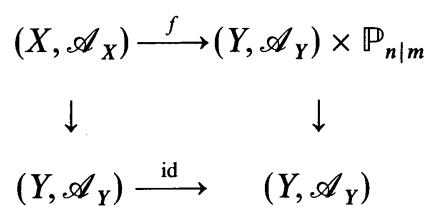

commutes. Then $\left(X, \mathscr{A}_{X}\right)$ is projective superalgebraic over $\mathscr{A}_{Y}$.

Proof. Let us first notice that this is true if $\left(X, \mathscr{A}_{X}\right)$ and $\left(Y, \mathscr{A}_{Y}\right)$ are just complex manifolds. For instance, if $X \subset Y \times \mathbb{P}_{n \mid m}$ has codimension one and $Y$ is Stein and contractible, the fact that $H^{1}\left(Y \times \mathbb{P}_{n}, \mathcal{O}\right)=0$ implies that the divisor line bundle of $X$ is a power of the hyperplane bundle; the case of higher codimension then follows over a sufficiently small subset of $Y$ by projecting to generic hyperplanes, and the general case follows by covering $Y$ with small Stein sets.

Let us now consider the general case. Let us remark that any section of $\mathcal{O}(d)$ on $Y \times \mathbb{P}_{n}$ is a homogeneous polynomial with coefficients depending on $Y$, and the analogous statement is true for $\mathscr{A}(d)$ on $\left(Y, \mathscr{A}_{Y}\right) \times \mathbb{P}_{n \mid m}$. By shrinking $Y$ as necessary we may assume that $X \subset Y \times \mathbb{P}_{n}$ is defined by a finite number of global sections of $\mathcal{O}(d)$, and we may also assume that $Y$ is Stein. We now may consider our sections as sections of $\mathscr{A}(d)$, and seek corrections terms so that these sections vanish on $\left(X, \mathscr{A}_{X}\right)$. As in the proof of Lemma 2, the obstructions to doing this are in $H^{1}\left(X, \mathscr{S}_{k} \otimes \mathcal{O}(d)\right)$ for a finite number of coherent analytic sheaves $\mathscr{S}_{k}$. Thus the cohomology of such sheaves will vanish on a fiber $X_{y}$ for $d$ large by the Grauert vanishing theorem, and by semicontinuity, we may take this vanishing to occur for every fiber $X_{y}$ by shrinking $Y$ as necessary. This being done, it follows that $H^{1}\left(X, \mathscr{S}_{k} \otimes \mathcal{O}(d)\right)=0$, as needed. We can thus extend our defining sections of $\mathcal{O}(d)$ to even global sections of $\mathscr{A}(d)$ over $\left(Y, \mathscr{A}_{Y}\right) \times \mathbb{P}_{n \mid m}$ which vanish on $\left(X, \mathscr{A}_{X}\right)$. 
We now ask for odd polynomials to adjoin to the above generators so as to generate the entire ideal of $\left(X, \mathscr{A}_{X}\right)$. We do this in the same manner as before. First we need some sections of $\mathscr{E} \otimes \mathcal{O}(d)$, where $\mathscr{E}$ is the characteristic sheaf of $\left(Y, \mathscr{A}_{Y}\right) \times \mathbb{P}_{n \mid m}$, which define $\left(X, \mathscr{A}_{X}\right)$ to first order, and then we wish to extend this to all orders. Again, the obstructions are in $H^{1}\left(X, \mathscr{S}_{k} \otimes \mathcal{O}(d)\right)$ for a finite number of coherent analytic sheaves $\mathscr{S}_{k}$. Taking $d$ to be large and $Y$ to be small, these cohomology groups vanish, and we are done.

\section{References}

1. Batchelor, M.: The structure of supermanifolds. Trans. Am. Math. Soc. 253, 329-338 (1979)

2. Berezin, F. A.: Method of second quantization. Pure and Applied Physics, vol. 24. NY: Academic Press 1966

3. Chow, W. L.: On Compact Complex Analytic Varieties. Am. J. Math. 71, 893-914 (1949)

4. Deligne, P.: Letter to Y.I. Manin

5. Eastwood, M., LeBrun, C.: Thickening and Supersymmetric Extensions of Complex Manifolds. Am. J. Math. 108, 1177-1192 (1986)

6. Friedan, D.: Notes on string theory and two-dimensional conformal Field Theory. EFI preprint 85-99 (1985)

7. Grauert, H.: Ein Theorem der analytischen Garben theorie and die Modulräume komplexer Strukturen. Publ. Inst. Hautes Études Scientifiques 5, 233-292 (1960)

8. Grauert, H.: Über Modifikationen und exzeptionelle analytische Mengen. Math. Ann. 146, 331-368 (1962)

9. Green, M. B., Schwarz, J. H., Witten, E.: Superstring Theory I \& II. Cambridge Monographs on Mathematical Physics. Cambridge, MA: Cambridge University Press 1987

10. Griffiths, P.: The extension problem in complex analysis II, embeddings with positive normal bundle. Am. J. Math. 88, 366-466 (1966)

11. Gunning, R.: Lectures on Riemann Surface. Princeton, NJ: Princeton University Press 1966

12. Michio Kaku: Introduction to SuperStrings. Berlin, Heidelberg, New York: Springer 1988

13. Kodaira, K.: On Kähler Varieties of Restricted Type (an Intrinsic Characterization of Algebraic Varieties). Ann. Math. 60, 28-48 (1954)

14. Kodaira, K., Spencer, D. C.: On deformation of complex analytic structure, III. Ann. Math. 71, 43-76 (1960)

15. LeBrun, C., Rothstein, M.: Moduli of super Riemann surfaces. Commun. Math. Phys. 117, 159-176 (1988)

16. Neveu, A., Schwartz, J. H.: Factorizable Dual Model of Pions. Nucl. Phys. B31, 86-112 (1971)

17. Manin, Y. I.: Gauge field theory and complex geometry. Berlin, Heidelberg, New York: Springer 1988

18. Mumford, D.: Stability of projective varieties. L'Ens. Math. 23, 39-110 (1977)

19. Penkov, I. B., Skornyakov, I. A.: Projectivity and $D$-affinity of Flag Supermanifolds. Russ. Math. Surv. 40, 233-234 (1987)

20. Ramond, P.: Dual theory of free fermions. Phys. Rev. D3, 2415-2418 (1971)

21. Rabin, J., Topiwala, P.: Super-Space GAGA and Moduli of SRS's. University of Chicago, preprint (1988)

22. Rothstein, M.: Deformation of complex supermanifolds. Proc. Am. Math. Soc. 95, 255-260 (1985)

23. Rothstein, M.: Integration on Non-compact Supermanifolds. Trans. Am. Math. Soc. 299, 387 (1987)

24. Wells, Jr, R. O.: Differential analysis on complex manifolds. Berlin, Heidelberg, New York: Springer 1982

25. Whitney, H.: The Self-intersections of a Smooth $n$-Manifold in $2 n$-Space. Ann. Math. 45, 247-293 (1944)

26. Wess, J., Zumino, B.: Supergauge transformations in four dimensions. Nucl. Phys. B70, 39-50 (1974)

Communicated by L. Alvarez-Gaumé

Received October 16, 1988; in revised form May 25, 1989 